\title{
EXISTENCE AND UNIQUENESS OF MILD SOLUTIONS FOR THE DAMPED BURGERS EQUATION IN WEIGHTED SOBOLEV SPACES ON THE HALF LINE
}

\author{
MOHAMMADREZA FOROUTAN* AND ALI EBADIAN \\ Department of Mathematics, Payame Noor University, P.O.Box 19395-3697, Tehran, Iran \\ *Corresponding author: foroutan_mohammadreza@yahoo.com

\begin{abstract}
This paper addresses an initial boundary value problem for the damped Burgers equation in weighted Sobolev spaces on half line. First, it introduces two normed spaces and present relations between them, which in turn enables us to analysis the existence and uniqueness of a local mild solution and of a global strong solution in these weighted spaces. The paper also studies the well-posedness of this equation in a semi-infinite interval.
\end{abstract}

\section{INTRODUCTION}

Nonlinear partial differential equations arise in a large number of mathematical and engineering problems $[1,2,6,7,17,25,27]$. A number of problems arising in science and engineering are set in semi-infinite domains, such as fluid flows in an infinite strip, nonlinear wave equations in quantum mechanics and so on. Burgers equation is one of the well-known equations in mathematics and physics, which is used to describe various kinds of phenomena such as mathematical model of turbulence [3] and the approximate theory of flow through a shock wave traveling in a viscous fluid [4].

In this work, we consider the space variable $x$ in $\Omega=[0, \infty)$, and the damped Burgers equation [20,23, 28] in the following form

$$
\varphi_{t}-\varphi_{x x}+\varphi \varphi_{x}+\lambda \varphi=0, \quad(x, t) \in \Omega \times \Omega,
$$

with the initial condition

$$
\varphi(x, 0)=\varphi_{0}(x), \quad x \in \Omega,
$$

2010 Mathematics Subject Classification. 35Q53, 35A01, 35A02.

Key words and phrases. damped Burgers equation; Hardy inequalities; mild solutions; semi-groups; strong solution. 
and the boundary conditions

$$
\varphi(0, t)=\lim _{x \rightarrow \infty} \varphi(x, t)=\lim _{x \rightarrow \infty} \varphi_{x}(x, t)=0, \quad t \in \Omega
$$

where $\lambda$ is positive constant. The analytical and numerical methods for solving (1.1) including the Pseudospectral methods [24], mixed finite difference and Galerkin methods [5], Taylor-Galerkin and Taylor-collocation methods [10], Chebyshev spectral collocation methods [15], modified extended backward differentiation formula [13] and modified Legendre rational spectral methods among others [19] have received much attention. For more details see $[11,12,16,21,26]$, among others. But, less attention has been paid to the study of existence and uniqueness of solution for Burgers equation.

Moreover, the study of solutions for the partial differential equations in bounded domains have achieved great success and popularity in recent years, while the study of solutions for the partial differential equations in unbounded domains have only received limited attention. In [8], Goubet and Shen studied the third-order $\mathrm{KdV}$ equation in a framework based on the dual-Petrov-Galerkin method on finite interval. Also in [14] Khanal et al. studied the fifth-order Kawahara equation in weighted Sobolev spaces on finite interval. This work deals with the existence and uniqueness of local mild solutions and of global strong solutions for the even-order equation posed on semi-infinite domains. Lu and $\mathrm{Li}$ [19] employed an algebraic mapping of the form $\omega(x)=\frac{x-1}{x+1}$ and proposed a set of Legendre rational functions which are orthogonal in $(0, \infty)$. In this paper, two normed spaces with the weight function $\chi(x)=\frac{1}{2} \frac{d}{d x} \omega(x)=\frac{1}{(x+1)^{2}}$ on the half line are introduced. Then the weak formulation of the linearized equation is considered:

$$
-\varphi_{x x}+\lambda \varphi=g
$$

The existence and uniqueness of solutions to the above formulation is established with any $g$ in these weighted spaces. Furthermore, by applying fixed-point argument we present the uniqueness of a local mild solution of (1.1) in semi-infinite interval.

The outline of this paper is as follows. Section 2 addresses, the special case of (1.1) in the form (1.4), then two normed spaces are introduced and their properties are investigated. Section 3 presents well-posedness results in the weighted spaces and describe the uniqueness of a local mild solution in these spaces. Finally, Section 4 addresses the existence of a global strong solution in weighted spaces.

\section{Hardy inequalities in Weighted Sobolev SPACES AND REPRESEntation of Bilinear Forms}

In this section, a weak formulation of the boundary value problem for the linear equation is presented as follows:

$$
\left\{\begin{array}{l}
-\varphi_{x x}+\lambda \varphi=g, \quad x \in \Omega \\
\varphi(0, t)=\lim _{x \rightarrow \infty} \varphi(x, t)=\lim _{x \rightarrow \infty} \varphi_{x}(x, t)=0
\end{array}\right.
$$


is presented. We first introduce some notations. Let $L^{p}(\Omega)$ with $p \in[1,+\infty]$ denote the usual Lebesgue space and $H^{k}(\Omega)$ the usual $L^{2}$-based space. Let $H_{0}^{k}(\Omega)$ denote the completion of $C_{0}^{\infty}(\Omega)$ under $H^{k}$-norm. Define

$$
\begin{aligned}
& \Phi(\Omega)=\left\{\varphi \in H_{0}^{1}(\Omega): \int_{\Omega} \varphi^{2}(x) \chi(x) d x<\infty\right\} . \\
& \Psi(\Omega)=\left\{\varphi \in \Phi(\Omega): \int_{\Omega} \varphi_{x}^{2}(x) \chi(x) d x<\infty\right\} .
\end{aligned}
$$

We define the bilinear form in $\Psi \times \Psi$ by

$$
b(\varphi, \phi)=\int_{\Omega} \varphi_{x}(x)(\phi(x) \chi(x))_{x} d x+\lambda \int_{\Omega} \varphi(x) \phi(x) \chi(x) d x,
$$

for any $\varphi$ and $\phi$ in $\Psi$. Thus, for any $g \in P$ the weak form of (2.1) is defined by

$$
b(\varphi, \phi)=(g, \phi)_{P}
$$

In addition, we write $P(\Omega)$ for $L_{\chi}^{2}(\Omega)$ and denote the inner product in $P$ by $(.,)_{P}$. We present the existence and uniqueness results for (2.1). For this purpose, we first present the following two Lemmas.

Lemma 2.1. The spaces $\Phi$ and $\Psi$ endowed with norm $\|\varphi\|_{L_{\chi}^{2}(\Omega)}$ and norm $\left\|\varphi_{x}\right\|_{L_{\chi}^{2}(\Omega)}$ are Hilbert spaces. The embedding relations $C_{0}^{\infty}(\Omega) \hookrightarrow \Psi \hookrightarrow \Phi$ are dense and continuous and the following Hardy type inequality holds:

$$
\begin{aligned}
& \int_{\Omega} \frac{\varphi^{2}(x)}{(x+1)^{4}} d x \leq \frac{1}{9} \int_{\Omega} \frac{\varphi_{x}^{2}(x)}{(x+1)^{2}} d x, \quad \forall \varphi \in \Phi . \\
& \int_{\Omega} \frac{\varphi^{2}(x)}{(x+1)^{2}} d x \leq \frac{64}{9} \int_{\Omega} \frac{\varphi_{x}^{2}(x)}{(x+1)^{2}} d x, \quad \forall \varphi \in \Psi
\end{aligned}
$$

Proof. It is clear that $\|\varphi\|_{L_{\chi}^{2}(\Omega)}$ and $\left\|\varphi_{x}\right\|_{L_{\chi}^{2}(\Omega)}$ are norms in $\Phi$ and $\Psi$, respectively. To show that $C_{0}^{\infty}(\Omega)$ is dense in $\Psi(\Omega)$, it suffices to show $C_{0}^{\infty}(\Omega)^{\perp}=0$. Let $\varphi \in C_{0}^{\infty}(\Omega)^{\perp}$. In this case, for any test function $\phi$, we have

$$
\int_{0}^{\infty} \varphi_{x}(x) \phi_{x}(x) \frac{1}{(x+1)^{2}} d x=0 .
$$

Then, straightway computations lead to $\varphi_{x}(x)=a(x+1)^{3}$. Since $\varphi_{x} \in L_{\chi}^{2}(\Omega)$, it can be concluded that $a=0$. Integrating once again, we get $\varphi(x)=b$. Then the boundary condition $\varphi(0)=0$ leads to $b=0$. This being so, $C_{0}^{\infty}(\Omega)^{\perp}=0$. In a similar manner, it can be shown that $C_{0}^{\infty}(\Omega)$ is dense in $\Phi(\Omega)$.

We now prove the Hardy inequality (2.6). Let $z \in I=(-1,1), x=\frac{1+z}{1-z}$ and $\psi(z)=\varphi(x)$. To prove the first inequality, it suffices to show that

$$
\int_{I} \psi^{2}(z)(1-z)^{2} d z \leq \frac{4}{9} \int_{I}\left(\partial_{z} \psi(z)\right)^{2}(1-z)^{4} d z
$$

Using $\psi(-1)=0$, for any $z \in I$ we have

$$
\psi^{2}(z)(1-z)^{3}=\int_{-1}^{z} \partial_{z}\left(\psi^{2}(z)(1-z)^{3}\right) d z .
$$


Therefore

$$
\begin{aligned}
\psi^{2}(z)(1-z)^{3}+ & 3 \int_{-1}^{z} \psi^{2}(z)(1-z)^{2} d z=2 \int_{-1}^{z} \psi(z) \partial_{z} \psi(z)(1-z)^{3} d z \\
& \leq 2\left(\int_{-1}^{z} \psi^{2}(z)(1-z)^{2} d z\right)^{\frac{1}{2}}\left(\int_{-1}^{z}\left(\partial_{z} \psi(z)\right)^{2}(1-z)^{4} d z\right)^{\frac{1}{2}}
\end{aligned}
$$

With letting $z \rightarrow 1$, we get (2.8). Moreover, we have

$$
\begin{aligned}
& \int_{\Omega} \frac{\varphi^{2}(x)}{(x+1)^{4}} d x=\frac{1}{8} \int_{I} \psi^{2}(z)(1-z)^{2} d z \\
& \int_{\Omega} \frac{\left(\partial_{x} \varphi(x)\right)^{2}}{(x+1)^{2}} d x=\frac{1}{2} \int_{I}\left(\partial_{z} \psi(z)\right)^{2}(1-z)^{4} d z
\end{aligned}
$$

Hence, by combining (2.8) and (2.9), we obtain the first result. To see that $\Psi \hookrightarrow \Phi$, one can apply (2.6),

$$
\|\varphi\|_{\Phi}^{2}=\int_{\Omega} \varphi^{2}(x) \frac{1}{(x+1)^{2}} d x \leq\|\varphi\|_{\Phi} \sup _{x \in \Omega}\left|\varphi(x) \frac{1}{x+1}\right| .
$$

The bound for $\sup _{x \in \Omega}\left|\varphi(x) \frac{1}{x+1}\right|$ can be obtained as follows.

$$
\begin{aligned}
& \varphi^{2}(x) \frac{1}{(x+1)^{2}}=2 \int_{0}^{x} \varphi(x) \varphi_{x}(x) \frac{1}{(x+1)^{2}} d x-2 \int_{0}^{x} \varphi^{2}(x) \frac{1}{(x+1)^{3}} d x \\
& \leq 2\left\|\varphi(x) \frac{1}{x+1}\right\|_{L^{2}}\left\|\varphi_{x}(x) \frac{1}{x+1}\right\|_{L^{2}}+2\left\|\varphi(x) \frac{1}{x+1}\right\|_{L^{2}}\left\|\varphi(x) \frac{1}{(x+1)^{2}}\right\|_{L^{2}} \\
& \leq \frac{8}{3}\|\varphi\|_{\Phi}\|\varphi\|_{\Psi} .
\end{aligned}
$$

Therefore

$$
\|\varphi\|_{\Phi} \leq \frac{8}{3}\|\varphi\|_{\Psi}
$$

Lemma 2.2. (Lax-Milgram Theorem) Assume that $W \subseteq V$ be two Hilbert spaces with continuous and dense embedding. Let $b(v, w)$ denote a bilinear form $V \times W$ and suppose that there exists $m, M>0$ such that

$$
\begin{array}{cl}
b(v, w) \leq M\|v\|_{V}\|w\|_{W}, & \forall v \in V, w \in W . \\
b(w, w) \geq m\|w\|_{W}^{2}, & \forall w \in W .
\end{array}
$$

Then for each $G \in V^{*}$ (the dual space of $V$ ), there exists $v \in V$ such that

$$
b(v, w)=G(w), \quad \forall w \in W
$$

This theorem is useful in showing the existence and uniqueness of a solution for a given differential equation. This general version of Lax-Milgram Theorem is due to Lions [18]; see also Lemma 4.4.4.1 in [9]. 
Theorem 2.1. Let $\lambda \geq 3$. Then, for each $g \in P$, there exists a unique solution $\varphi \in \Psi$ such that, for all $\phi \in \Psi$,

$$
b(\varphi, \phi)=(g, \phi)_{P}
$$

Therefore, we able define an operator $S: D(S) \rightarrow P$ given by

$$
S \varphi=g
$$

where $D(S)=\{\varphi \in \Psi, S \varphi \in P\}$.

Proof. For $\varphi \in \Psi$ and $\phi \in \Psi$, we check that $b(\varphi, \phi)$ as defined in (2.4) satisfies the conditions set in Lemma 2.2. Hence, form (2.4) we can write

$$
b(\varphi, \phi)=\int_{\Omega} \varphi_{x}(x) \phi_{x}(x) \chi(x) d x+\int_{\Omega} \varphi_{x}(x) \phi(x) \chi^{\prime}(x) d x+\lambda \int_{\Omega} \varphi(x) \phi(x) \chi(x) d x .
$$

By using (2.6) and (2.7), the various terms of (2.15) on the right can be written in the following way.

$$
\begin{aligned}
\int_{\Omega} \varphi_{x}(x) \phi_{x}(x) \chi(x) d x & \leq\left(\int_{\Omega} \frac{\varphi_{x}^{2}(x)}{(x+1)^{2}} d x\right)^{\frac{1}{2}}\left(\int_{\Omega} \frac{\phi_{x}^{2}(x)}{(x+1)^{2}} d x\right)^{\frac{1}{2}}=\|\varphi\|_{\Psi}\|\phi\|_{\Psi} . \\
\int_{\Omega} \varphi_{x}(x) \phi(x) \chi^{\prime}(x) d x & \leq 2\left(\int_{\Omega} \frac{\varphi_{x}^{2}(x)}{(x+1)^{2}} d x\right)^{\frac{1}{2}}\left(\int_{\Omega} \frac{\phi^{2}(x)}{(x+1)^{4}} d x\right)^{\frac{1}{2}} \\
& \leq \frac{2}{3}\left(\int_{\Omega} \frac{\varphi_{x}^{2}(x)}{(x+1)^{2}} d x\right)^{\frac{1}{2}}\left(\int_{\Omega} \frac{\phi_{x}^{2}(x)}{(x+1)^{2}} d x\right)^{\frac{1}{2}}=\frac{2}{3}\|\varphi\|_{\Psi}\left\|_{\phi}\right\|_{\Psi} . \\
\int_{\Omega} \varphi(x) \phi(x) \chi(x) d x & \leq\left(\int_{\Omega} \frac{\varphi^{2}(x)}{(x+1)^{2}} d x\right)^{\frac{1}{2}}\left(\int_{\Omega} \frac{\phi^{2}(x)}{(x+1)^{2}} d x\right)^{\frac{1}{2}} \\
& \leq \frac{64}{9}\left(\int_{\Omega} \frac{\varphi_{x}^{2}(x)}{(x+1)^{2}} d x\right)^{\frac{1}{2}}\left(\int_{\Omega} \frac{\phi_{x}^{2}(x)}{(x+1)^{2}} d x\right)^{\frac{1}{2}}=\frac{64}{9}\|\varphi\|_{\Psi}\|\phi\|_{\Psi} .
\end{aligned}
$$

Combining these three results, we have

$$
b(\varphi, \phi) \leq\left(\frac{5}{3}+\frac{64}{9} \lambda\right)\|\varphi\|_{\Psi}\|\phi\|_{\Psi}
$$

In order to prove the weak coercivity (2.11), for all $\varphi \in \Psi$ we have

$$
\begin{aligned}
b(\varphi, \varphi) & =\int_{\Omega} \varphi_{x}^{2}(x) \chi(x) d x+\int_{\Omega} \varphi_{x}(x) \varphi(x) \chi^{\prime}(x) d x+\lambda \int_{\Omega} \varphi^{2}(x) \chi(x) d x \\
& =\int_{\Omega} \frac{\varphi_{x}^{2}(x)}{(x+1)^{2}} d x-3 \int_{\Omega} \frac{\varphi^{2}(x)}{(x+1)^{3}} d x+\lambda \int_{\Omega} \frac{\varphi^{2}(x)}{(x+1)^{2}} d x \\
& \geq \int_{\Omega} \frac{\varphi_{x}^{2}(x)}{(x+1)^{2}} d x=\|\varphi\|_{\Psi}^{2} .
\end{aligned}
$$

Thus, by the Lemma 2.2 , there exists $\varphi \in \Psi$ such that $b(\varphi, \phi)$ defines a linear functional $G$ on $\Psi$ :

$$
G(\phi)=b(\varphi, \phi)
$$


From (2.16),

$$
\|G\|_{\Psi^{*}}=\sup _{\phi \in \Psi} \frac{\|G(\phi)\|}{\|\phi\|_{\Psi}} \leq \alpha\|\varphi\|_{\Psi}<\infty
$$

Hence, the functional $G$ is continuous. Thanks to Riesz Representation Theorem, there exists a unique $g$ such that $G(\phi)=(g, \phi)_{P}$. Since, $g$ depends on $\varphi$, so we can write this dependence as $S \varphi=g$, where $S: D(S) \rightarrow \Phi$ is a linear operator. Therefore

$$
G(\phi)=b(\varphi, \phi)=(S \varphi, \phi)_{P}
$$

and $\|S \varphi\|_{P} \leq M\|\varphi\|_{\Psi}$ which means that $S$ is continuous. Also we have

$$
\|S \varphi\|_{P}=\sup _{\phi \in \Psi} \frac{\|b(\varphi, \phi)\|}{\|\phi\|_{P}} \geq \sup _{\phi \in \Psi} \frac{\|b(\varphi, \phi)\|}{\|\phi\|_{\Psi}} \geq \beta_{1}\|\varphi\|_{\Psi} .
$$

Hence, $S$ is bounded below. We now show that the range $R(S)$ of $S$ is closed. Indeed, if $\left\{S \varphi_{n}\right\} \in P$ is a Cauchy sequence then so is $\left\{\varphi_{n}\right\} \in \Psi$ and by (2.20) we obtain

$$
\left\|\varphi_{m}-\varphi_{n}\right\|_{\Psi} \leq \beta_{2}\left\|S\left(\varphi_{m}-\varphi_{n}\right)\right\|_{P}=\beta_{2}\left\|S \varphi_{m}-S \varphi_{n}\right\|_{P}
$$

So that $\left\{\varphi_{n}\right\}$ converges to some $\varphi \in \Psi$. Since $S$ is continuous, $\left\{S \varphi_{n}\right\}$ converges to $S \varphi$ which proves that $R(S)$ is closed. Furthermore, we show that $S$ is surjective. If this was not true, there exists a $\varphi_{0} \in R(S)^{\perp}$ with $\varphi_{0} \neq 0$ such that $\left(S \varphi, \varphi_{0}\right)_{P}=b\left(\varphi, \varphi_{0}\right)=0$ for each $\varphi \in \Psi$. But this is contradiction with (2.4). Thus $\varphi_{0}=0$ and $R(S)=P$. Using $(2.20)$ we get

$$
\sup _{\varphi \in \Psi} \frac{\|b(\varphi, \phi)\|}{\|\varphi\|_{\Psi}}=\sup _{\varphi \in \Psi} \frac{\left\|(S \varphi, \phi)_{P}\right\|}{\|\varphi\|_{\Psi}} \geq \sup _{\varphi \in \Psi} \frac{\left\|(S \varphi, \phi)_{P}\right\|}{\beta_{1}^{-1}\|S \varphi\|_{P}}=\beta_{1} \sup _{\psi \in \Psi} \frac{\left\|(\psi, \phi)_{P}\right\|}{\|\psi\|_{P}}=\beta_{1}\|\phi\|_{P} .
$$

We now show that there is at most one element $\varphi \in \Psi$ satisfying (2.17). Assume the contrary, i.e., that $b\left(\varphi_{1}, \phi\right)=G(\phi)$ and $b\left(\varphi_{2}, \phi\right)=G(\phi)$ for all $\phi \in \Psi$. Then by linearity,

$$
b\left(\varphi_{1}-\varphi_{2}, \phi\right)=0, \quad \forall \phi \in \Psi .
$$

According to Lemma 2.1, as $C_{0}^{\infty}(\Omega)$ is densely embedded in $\Psi$, there is a sequence $\varphi_{n} C_{0}^{\infty}(\Omega)$ such that

$$
\varphi_{n} \rightarrow \varphi_{1}-\varphi_{2}, \quad \text { in } \Psi
$$

From (2.21),

$$
\begin{aligned}
\left\|\varphi_{n}\right\|_{\Psi}^{2} & \leq b\left(\varphi_{n}, \varphi_{n}\right)=b\left(\varphi_{1}-\varphi_{2}, \varphi_{n}\right)+b\left(\varphi_{n}-\left(\varphi_{1}-\varphi_{2}\right), \varphi_{n}\right) \\
& \leq M\left\|\varphi_{n}-\left(\varphi_{1}-\varphi_{2}\right)\right\|_{\Psi}\left\|\varphi_{n}\right\|_{\Psi} .
\end{aligned}
$$

Letting $n \rightarrow \infty$, one has $\varphi_{n} \rightarrow 0$ in $\Psi$ and consequently

$$
\left\|\varphi_{1}-\varphi_{2}\right\|_{\Psi} \leq\left\|\varphi_{n}\right\|_{\Psi}+\left\|\varphi_{n}-\left(\varphi_{1}-\varphi_{2}\right)\right\|_{\Psi} \rightarrow 0 \text { as } n \rightarrow \infty .
$$

Therefore, $\varphi_{1}=\varphi_{2}$ in $\Psi$. 


\section{The DAmped Burgers equation}

In this section, we consider the damped Burgers equation (1.1) with initial and boundary values (1.2) and (1.3). We study the mild solutions of this equation. To this end, we show that $-S$ is an infinitesimal generator of a semi-group where $S$ is defined in Theorem 2.1. Using classical theory of linear semi-group and the Hille-Yosida Theorem as presented in [22] we establish the following theorem for the operator $S$.

Theorem 3.1. Let $P, S$ and $D(S)$ be defined as in the previous section. Then operator $-S$ in the infinitesimal generator of a semi-group of contraction $e^{-t S}$ in $P$.

Proof. According to the Hille-Yosida Theorem it is sufficient to check that $S$ is closed, $D(S)$ is dense in $P$ and $\left\|(\lambda-S)^{-1} g\right\|_{P} \leq \frac{1}{\lambda}\|g\|_{P}$ for any $\lambda>0$. From (2.21) it follows that $S^{-1}$ is one to one. Thus, $S$ is closed. $D(S)$ is dense in $P$, since $C_{0}^{\infty}(\Omega) \subset D(S)$.

Let $\varphi=(\lambda+S)^{-1} g$ where $g \in P$. Then $(\lambda+S) \varphi=g$ and

$$
(g, \varphi)_{P}=((\lambda+S) \varphi, \varphi)_{P}=\lambda\|\varphi\|_{P}^{2}+(S \varphi, \varphi)_{P}
$$

From (2.20) it immediately follows that $(S \varphi, \varphi)_{P} \geq 0$. Then by (3.1) we have

$$
\|\varphi\|_{P} \leq \frac{1}{\lambda}\|g\|_{P}
$$

Before studying the mild solution, lets define the bilinear form on $\Psi \times \Psi$ as

$$
A(\varphi, \phi)=(\varphi \phi)_{x} \quad(\varphi, \phi) \in \Psi \times \Psi
$$

A mild solution of the initial boundary value problem $(1.1)$ is a function $\varphi \in H=C([0, T] ; P) \cap L^{2}(0, T ; \Psi)$ satisfying

$$
\left\{\begin{array}{l}
\frac{d \varphi}{d t}+S \varphi=-A(\varphi, \varphi) \\
\varphi(0)=\varphi_{0}
\end{array}\right.
$$

where $\varphi_{0} \in P$ and $T>0$. Let $K(t)=e^{-t S}$ where $S$ is defined in the previous section. We plan to apply a fixed-point argument to the integral equation

$$
\varphi(t)=K(t) \varphi_{0}-\frac{1}{2} \int_{0}^{t} K(t-\tau) A(\varphi, \varphi)(\tau) d \tau .
$$

For this purpose, we show that if $T$ is small enough, then

$$
\varphi(t) \rightarrow F(\varphi(t))
$$

is a contraction $H$, where the right side of (3.4) is denoted by $F(\varphi(t))$. 
Theorem 3.2. For any given $\varphi_{0} \in P$, there exists $T>0$ such that the problem (1.1) has a unique mild solution in $H$. Furthermore, the following energy identity holds for all $t \geq 0$ :

$$
\|\varphi(t)\|_{P}^{2}+\int_{0}^{t}\|\varphi(\tau)\|_{\Psi}^{2} d \tau \leq\left\|\varphi_{0}\right\|_{P}^{2}+C \int_{0}^{t}\|\varphi(\tau)\|_{L^{2}}^{2}\|\varphi(\tau)\|_{P}\|\varphi(\tau)\|_{\Psi} d \tau .
$$

Proof. We first prove that for any $(\varphi, \phi) \in \Psi \times \Psi$,

$$
\|A(\varphi, \phi)\|_{\Psi^{*}} \leq C\|\varphi\|_{L^{2}}\|\phi\|_{P}^{\frac{1}{2}}\|\phi\|_{\Psi}^{\frac{1}{2}}
$$

Let $\psi \in \Psi$, one obtains by integrating by parts

$$
\begin{aligned}
(A(\varphi, \phi), \psi)_{P} & =\int_{\Omega}(\varphi(x) \phi(x))_{x} \psi(x) \chi(x) d x \\
& =-\int_{\Omega} \varphi(x) \phi(x) \psi_{x}(x) \chi(x) d x-\int_{\Omega} \varphi(x) \phi(x) \psi(x) \chi^{\prime}(x) d x .
\end{aligned}
$$

By using Cauchy-Schwarz inequality to bound the first term on the right-hand side of this equality, we have

$$
\begin{aligned}
\int_{\Omega} \varphi(x) \phi(x) \psi_{x}(x) \chi(x) d x= & \int_{\Omega} \varphi(x) \phi(x) \frac{1}{x+1} \psi_{x}(x) \frac{1}{x+1} d x \\
& \leq\|\varphi(x)\|_{L^{2}}\|\psi(x)\|_{\Psi} \sup _{x \in \Omega}\left|\phi(x) \frac{1}{x+1}\right| .
\end{aligned}
$$

To bound $\sup _{x \in \Omega}\left|\phi(x) \frac{1}{x+1}\right|$, apply (2.6) in Lemma 2.1 to obtain

$$
\begin{aligned}
& \phi^{2}(x) \frac{1}{(x+1)^{2}}=2 \int_{0}^{x} \phi(x) \phi_{x}(x) \frac{1}{(x+1)^{2}} d x-2 \int_{0}^{x} \phi^{2}(x) \frac{1}{(x+1)^{3}} d x \\
& \leq 2\left\|\phi(x) \frac{1}{(x+1)}\right\|_{L^{2}}\left\|\phi_{x}(x) \frac{1}{(x+1)}\right\|_{L^{2}}+2\left\|\phi(x) \frac{1}{(x+1)}\right\|_{L^{2}}\left\|\phi(x) \frac{1}{(x+1)^{2}}\right\|_{L^{2}} \\
& \quad \leq C\|\phi(x)\|_{P}\|\phi(x)\|_{\Psi} .
\end{aligned}
$$

Therefore

$$
\int_{\Omega} \varphi(x) \phi(x) \psi_{x}(x) \chi(x) d x \leq C\|\varphi(x)\|_{L^{2}}\|\phi(x)\|_{P}^{\frac{1}{2}}\|\phi(x)\|_{\Psi}^{\frac{1}{2}}\|\psi(x)\|_{\Psi}
$$

By using (2.6) in Lemma 2.1, the second term in (3.8) can be bounded similarly.

$$
\begin{aligned}
\int_{\Omega} \varphi(x) \phi(x) \psi(x) \chi^{\prime}(x) d x= & -2 \int_{\Omega} \varphi(x) \phi(x) \frac{1}{x+1} \psi(x) \frac{1}{(x+1)^{2}} d x \\
& \leq 3\|\varphi(x)\|_{L^{2}}\left\|\psi(x) \frac{1}{(x+1)}\right\|_{L^{2}} \sup _{x \in \Omega}\left|\phi(x) \frac{1}{x+1}\right| \\
& \leq C\|\varphi(x)\|_{L^{2}}\|\phi(x)\|_{P}^{\frac{1}{2}}\|\phi(x)\|_{\Psi}^{\frac{1}{2}}\|\psi(x)\|_{\Psi} .
\end{aligned}
$$

Hence, by inserting (3.10) and (3.11) into (3.8), we obtain (3.7). Similar process show that

$$
\|A(\varphi, \phi)\|_{\Psi^{*}} \leq C\|\phi\|_{L^{2}}\|\varphi\|_{P}^{\frac{1}{2}}\|\varphi\|_{\Psi}^{\frac{1}{2}}
$$

We now prove that

$$
\|\varphi\|_{L^{2}} \leq\left\|\varphi_{0}\right\|_{L^{2}}
$$


Taking the $L^{2}$-inner product of $(1.1)$ with $\varphi(x)$ over the interval $(0, \infty)$, we obtain

$$
\int_{\Omega} \varphi(x) \varphi_{t}(x) d x-\int_{\Omega} \varphi(x) \varphi_{x x}(x) d x+\int_{\Omega} \varphi^{2}(x) \varphi_{x}(x) d x=0 .
$$

Using mainly integration by parts, we can write the various terms as

$$
\begin{aligned}
\int_{\Omega} \varphi(x) \varphi_{t}(x) d x & =\frac{1}{2} \int_{\Omega} \frac{d}{d t} \varphi^{2}(x) d x=\frac{1}{2} \frac{d}{d t} \int_{\Omega} \varphi^{2}(x) d x=\frac{1}{2} \frac{d}{d t}\|\varphi(x)\|_{L^{2}}^{2} . \\
-\int_{\Omega} \varphi(x) \varphi_{x x}(x) d x & =\int_{\Omega} \varphi_{x}^{2}(x) d x=\left\|\varphi_{x}(x)\right\|_{L^{2}}^{2} . \\
\int_{\Omega} \varphi^{2}(x) \varphi_{x}(x) d x & =0 .
\end{aligned}
$$

Substituting (3.15)-(3.17) into (3.14), we obtain

$$
\frac{d}{d t}\|\varphi(x)\|_{L^{2}}^{2}=-2\left\|\varphi_{x}(x)\right\|_{L^{2}}^{2}
$$

Using the Poincare inequality on the right-hand side of inequality (3.18), we attain

$$
\frac{d}{d t}\|\varphi(x)\|_{L^{2}}^{2}=-2\left\|\varphi_{x}(x)\right\|_{L^{2}}^{2} \leq-2\|\varphi(x)\|_{L^{2}}^{2} .
$$

Integrating inequality (3.19) with respect to time from 0 to $t$, we get

$$
\|\varphi(x)\|_{L^{2}} \leq e^{-t}\left\|\varphi_{0}(x)\right\|_{L^{2}}
$$

Since $\varphi_{0} \in L^{2}(\Omega)$ and $t \in[0, T]$,then (3.13) is achieved. Now, let us introduce the Banach space $P$ with norm

$$
\|\varphi\|_{H}=\sup _{t \in[0, T]}\|\varphi\|_{P}+\|\varphi\|_{L^{2}(0, T ; \Psi)}
$$

Let $D=2\left\|\varphi_{0}\right\|_{L^{2}}$ and $B_{D}=\left\{\varphi \in H:\|\varphi\|_{H} \leq D\right\}$. We now prove that if $T$ is small enough, then $F$ maps $B_{D}$ into itself. Let $\varphi \in B_{D}$ and $F(\varphi)$ satisfies

$$
\frac{d}{d t} F(\varphi)+S F(\varphi)=-A(\varphi, \varphi)
$$

Hence

$$
\frac{d}{d t}\|F(\varphi)\|_{P}^{2}+2 b(F(\varphi), F(\varphi))=-2(A(\varphi, \varphi), F(\varphi))_{P}
$$

According to the proof of Theorem 2.1, we get

$$
2 b(F(\varphi), F(\varphi)) \geq 2\|F(\varphi)\|_{\Psi}^{2}
$$

By using (3.7),

$$
\begin{aligned}
2\left|(A(\varphi, \varphi), F(\varphi))_{P}\right| \leq & 2\|A(\varphi, \varphi)\|_{\Psi^{*}}\|F(\varphi)\|_{\Psi} \\
& \leq 3\|F(\varphi)\|_{\Psi}^{2}+C\|\varphi\|_{L^{2}}^{2}\|\varphi\|_{P}\|\varphi\|_{\Psi}
\end{aligned}
$$


Therefore

$$
\|F(\varphi)\|_{P}^{2}+\int_{0}^{t}\|F(\varphi)\|_{\Psi}^{2} d \tau \leq\left\|\varphi_{0}\right\|_{P}^{2}+C \int_{0}^{t}\|\varphi(\tau)\|_{L^{2}}^{2}\|\varphi(\tau)\|_{P}\|\varphi(\tau)\|_{\Psi} d \tau .
$$

If we choose $T>0$ such that $D^{2}+8 C \sqrt{T} D^{4}<D^{2}$, then

$$
\|F(\varphi)\|_{H}<D
$$

To show $F$ is a contraction, first note that

$$
F(\varphi)-F(\phi)=-\int_{0}^{t} K(t-\tau)(A(\varphi-\phi, \varphi)+A(\phi, \varphi-\phi)) d \tau .
$$

Using once again (3.7) and (3.12) for $A(\varphi-\phi, \varphi)$ and $A(\phi, \varphi-\phi)$, respectively

$$
\begin{aligned}
& \|F(\varphi)-F(\phi)\|_{P}^{2}+\int_{0}^{t}\|F(\varphi)-F(\phi)\|_{\Psi}^{2} \\
& \leq \int_{0}^{t}\left(\|\varphi\|_{L^{2}}^{2}\|\varphi-\phi\|_{P}\|\varphi-\phi\|_{\Psi}+\|\phi\|_{L^{2}}^{2}\|\varphi-\phi\|_{P}\|\varphi-\phi\|_{\Psi}\right) d \tau \\
& \leq C \sqrt{T}\left(\|\varphi\|_{L^{2}}^{2}+\|\phi\|_{L^{2}}^{2}\right)\|\varphi-\phi\|_{H}^{2} .
\end{aligned}
$$

If $T$ is further restricted to $\delta^{2}=2 C \sqrt{T}<1$, then

$$
\|F(\varphi)-F(\phi)\|_{H} \leq \delta\|\varphi-\phi\|_{H}
$$

Applying the contraction mapping principle completes the proof of the theorem.

\section{Strong SOlutions}

In this section, we study solutions of the boundary initial value problem (1.1) in a stronger sense and establish the global existence and uniqueness of such solutions.

Theorem 4.1. Let $T_{0}>0$ and $\varphi_{0} \in P(\Omega)$ be given. Then there exists $T \in\left(0, T_{0}\right]$ such that it possesses a unique solution

$$
\varphi \in C([0, T] ; P) \cap L^{2}(0, T ; \Psi) .
$$

Moreover, if there exist a constant $C=C(T)>0$ such that

$$
\left\|\varphi_{0}\right\|_{L^{2}(\Omega)} \leq C
$$

then the problem (1.1) has a strong solution on $[0, T]$.

Proof. For $\varphi_{0} \in P$, Theorem 3.2 assures that (1.1) admits the unique solution

$$
\varphi \in C([0, T] ; P) \cap L^{2}(0, T ; \Psi)
$$

For the global existence, we only have to show that the solution cannot blow up at any finite time $T$. For this purpose, we apply (3.13) to show that, for $t \in[0, T]$,

$$
\|\varphi(t)\|_{P} \leq C(T)\left\|\varphi_{0}\right\|_{P}
$$


Taking the $H$-inner product of $(1.1)$ with $\varphi$ over the interval $(0, \infty)$, we get

$$
\int_{\Omega} \varphi(x) \varphi_{t}(x) \chi(x) d x-\int_{\Omega} \varphi(x) \varphi_{x x}(x) \chi(x) d x+\int_{\Omega} \varphi^{2}(x) \varphi_{x}(x) \chi(x) d x+\lambda \int_{\Omega} \varphi^{2}(x) \chi(x) d x=0 .
$$

The terms on the left can be written as

$$
\begin{array}{r}
-\int_{\Omega} \varphi(x) \varphi_{x x}(x) \chi(x) d x=\int_{\Omega} \varphi_{x}^{2}(x) \chi(x) d x+\int_{\Omega} \varphi(x) \varphi_{x}(x) \chi^{\prime}(x) d x \\
=\|\varphi\|_{\Psi}^{2}-\frac{1}{2} \int_{\Omega} \varphi^{2}(x) \chi^{\prime \prime}(x) d x=\|\varphi\|_{\Psi}^{2}-3 \int_{\Omega} \varphi^{2}(x) \frac{1}{(x+1)^{4}} d x . \\
\int_{\Omega} \varphi^{2}(x) \varphi_{x}(x) \chi(x) d x=-\frac{1}{3} \int_{\Omega} \varphi^{3}(x) \chi^{\prime}(x) d x=\frac{2}{3} \int_{\Omega} \varphi^{3}(x) \frac{1}{(x+1)^{3}} d x .
\end{array}
$$

Substituting (4.5) and (4.6) into (4.4), we obtain

$$
\frac{d}{d t}\|\varphi\|_{P}^{2}+\|\varphi\|_{\Psi}^{2}=3 \int_{\Omega} \varphi^{2}(x) \frac{1}{(x+1)^{4}} d x-\frac{2}{3} \int_{\Omega} \varphi^{3}(x) \frac{1}{(x+1)^{3}} d x-\lambda \int_{\Omega} \varphi^{2}(x) \frac{1}{(x+1)^{2}} d x .
$$

The terms on the right-hand can be bounded as

$$
\begin{gathered}
\int_{\Omega} \varphi^{2}(x) \frac{1}{(x+1)^{4}} d x \leq \int_{\Omega} \varphi^{2}(x) \frac{1}{(x+1)^{2}} d x=\|\varphi\|_{P}^{2} . \\
\int_{\Omega} \varphi^{3}(x) \frac{1}{(x+1)^{3}} d x \leq \int_{\Omega} \varphi^{3}(x) \frac{1}{(x+1)^{2}} d x=\int_{\Omega} \varphi(x) \varphi^{2}(x) \frac{1}{(x+1)^{2}} d x \\
\leq \frac{1}{2}\|\varphi\|_{P}^{2}+\frac{1}{2}\|\varphi\|_{L^{2}}^{2}\|\varphi\|_{P}^{2} \leq \frac{1}{2}\|\varphi\|_{P}^{2}+\frac{1}{2}\left\|\varphi_{0}\right\|_{L^{2}}^{2}\|\varphi\|_{P}^{2} .
\end{gathered}
$$

Inserting (4.8) and (4.9) into (4.7), we have

$$
\frac{d}{d t}\|\varphi\|_{P}^{2}+\|\varphi\|_{\Psi}^{2}-\left(\frac{10}{3}\|\varphi\|_{P}^{2}+\frac{1}{3}\left\|\varphi_{0}\right\|_{L^{2}}^{2}\|\varphi\|_{P}^{2}+\lambda\|\varphi\|_{P}^{2}\right) \leq 0
$$

On the other hand, since $\|\varphi\|_{P} \leq\|\varphi\|_{\Psi}$, we conclude that

$$
\left.\frac{d}{d t}\|\varphi\|_{P}^{2}-\left(\frac{7}{3}+\frac{1}{3}\left\|\varphi_{0}\right\|_{L^{2}}^{2}+\lambda\right)\|\varphi\|_{P}^{2}\right) \leq 0
$$

Applying the Gronwall's inequality completes the proof of this theorem.

\section{REFERENCES}

[1] R. Abazari, Application of Extended Tanh Function Method on KdV-Burgers Equation with Forcing Term, Rom. J. Phys. 59(1-2) (2014), 3-11.

[2] A. Biswas, S. Kumar, E.V. Krishnan, B. Ahmed, A. Strong, S. Johnson, A. Yildirim Topological Solitons and Other Solutions to Potential KdV Equation, Rom. J. Phys. 65(4) (2013), 1125-1137.

[3] J.M. Burgers, A mathematical model illustrating the theory of turbulence, Adv. Appl. Mech. 1 (1948), 171-199.

[4] J.D. Cole, On a puasilinear parabolic equations occurring in aerodynamics, Quart. Appl. Math. 9 (1951), 225-236.

[5] M. Dehghan, B.N. Saray and M. Lakestani, Mixed finite difference and Galerkin methods for solving Burgers equations using interpolating scaling functions, Math. Meth. Appl. Sci. 37 (6) (2014), 894-912.

[6] G. Ebadi, A. Mojaver, H. Triki, A. Yildirim, A. Biswas, Topological solitons and other solutions of the RosenauCKdV equation with power law nonlinearity, Rom. J. Phys. 58 (2013), 3-14. 
[7] G. Ebadi, N. Yousefzadeh, H. Triki, A. Yildirim, A. Biswas, Envolope solitons, peridic waves and other solutions to BoussinesqCBurgers equstion, Rom. J. Phys. 64 (2012), 915-932.

[8] D. Goubet and J. Shen, On the dual Petrov-Galerkin formulation of the KdV equation on a finite interval, Adv. Diff. Equs. 12 (2007), 221-239.

[9] P. Grisvard, El liptic problems in nonsmooth domains, Volume 24 of monographs and studies in Mathematics, Pitman (Advanced Publishing Program), Boston, MA (1985).

[10] D. Idris, C.I. Aynur and S. Ali, Taylor Galerkin and Taylor collocation methods for the numerical solutions of Burgers equation using B-splines, Commun. Nonlinear Sci. Numer. Simul. 16:7 (2011), 2696-2708.

[11] I.E. Inan, D. Kaya and Y. Ugurlu, Auto Backlund transformation and similarity reductions for coupled Burgers equation, Appl. Math. Comput. 216 (2010), 2507-2511.

[12] K. Ismail and S. Ibrahim, An efficient computational method for the optimal control problem for the Burgers equation, Math. Comput. Model. 44 (2006), 973-982.

[13] M. Javidi, A numerical solution of Burgers equation based on modified extended BDF scheme, Int. Math. Forum 1,(2006), $1565-1570$.

[14] N. Khanal, J. Wu and J.M. Yuan, The Kawahara equation in weighted Sobolev spaces, Nonlinearity 21 (2008), $1489-1505$.

[15] A.H. Khater, R.S. Temsah and M.M. Hassan, A Chebyshev spectral collocation method for solving Burgers-type equation, J. Comput. Appl. Math. 222 (2008), 333-350.

[16] M. K. Kunisch and S. Volkwein, Control of the Burgers equation by a reduced-order approach using proper orthogonal decomposition, J. Optim. Theory Appl. 102 (1999), 345-371.

[17] Jerome L.V. Lewandowski, A marker method for the solution of the damped Burgers equation, Numer. Methods Partial Differ. 22(1) (2005), 48-68.

[18] J.L. Lions, Sur les problems aux limites du type derive oblique, Ann. Math. 64 (1956), 207-239.

[19] S. Lu and M. Li, Modified Legendre rational spectral method for thr Burgers equation on the half line, Int. J. Comput. Math. 85:6 (2008), 865-875.

[20] W. Malfliet, Approximate solution of the damped Burgers equation, J. Phys. A: Math. Gen. 26 (1993), L723-L728.

[21] R.C. Mittal and G. Arora, Numerical solution of the coupled viscous Burgers equation, Commun. Nonlinear Sci. Numer. Simul. 16 (2011), 1304-1313.

[22] A. Pazy, Semigroups of linear operators and applications to partial differential equations, New York: Springer, (1983).

[23] Y. Peng, W. Chen, A new similarity solution of the Burgers equation with linear damping Czech, J, Phys. 56 (2008) $317-428$.

[24] Z. Sabeh, M. Shamsi and M. Dehghan, Distributed optimal control of the viscous Burgers equation via a Legendre pseudospectral approach, Math. Meth. Appl. Sci. 39(12) (2015),3350-3360

[25] H. Triki, A. Yildirim, T. Hayat, O.M. Aldossary, A. Biswas, Topological and non-topological solitons of a generalized derivative nonlinear Schrodingers equation with perturbation terms, Rom. J. Phys. 64 (2012), 672-684.

[26] F. Yilmaz and B. Karasozen, Solving optimal control problems for the unsteady Burgers equation in COMSOL multiphysics, J. Comput. Appl. Math. 235 (16) (2011), 4839-4850.

[27] N.Y. Fard, M.R. Foroutan, M. Eslami, M. Mirzazadeh, A. Biswas, Solitary waves and other solutions to KadomtsevPetviashvili equation with spatio-temporal dispersion, Rom. J. Phys. 60 (2015), 1337-1360.

[28] B. M. Vaganan, M. S. Kumaran, Kummer function solutions of damped Burgers equations with time-dependent viscosity by exact linearization, Nonlinear Anal. Real World Appl. 4 (2003), 723-741. 